\title{
Estudio de la Legalidad Brasileña de las Escuelas Públicas Municipales en Cuanto a la Promoción de la Alimentación Sana para el Control y Prevención de la Salud Oral en el Contexto de las Enferme- dades Gastrointestinales Inflamatorias
}

\author{
Study of the Brazilian Legality of the Municipal Public Schools Regarding the \\ Promotion of Healthy Eating for Control and Prevention of Oral Health in the Context \\ of Inflammatory Intestinal Diseases
}

Jessica Rayssa Teixeira1; Maria Georgina Farias'; Jamili Raiane Campos; Rayla Kenia Santos ${ }^{1}$; Thayná Almeida²; Ana Cássia Reis ${ }^{2,3}$; Pablo Maranhão4 \& Kalena Maranhão

TEIXEIRA, J. R.; FARIAS, M. G.; CAMPOS, J. R.; SANTOS, R. K.; ALMEIDA, T.; REIS, A. C.; MARANHÃO, P. \& MARANHÃO, K. Estudio de la legalidad brasileña de las escuelas públicas municipales en cuanto a la promoción de la alimentación sana en el control y prevención a la salud oral en el contexto de las Enfermedades Gastrointestinales Inflamatorias. Int. J. Odontostomat., 13(3):363-366, 2019.

RESUMEN: Las enfermedades gastrointestinales inflamatorias se caracterizan por la desarmonía de la flora bacteriana del intestino. Estos cambios se caracterizan como un problema de salud pública que demuestra que las principales causas de las enfermedades están asociadas a la alimentación inadecuada y, como consecuencia de cambios en la mucosa oral. Los países desarrollados están actualmente definiendo estrategias para el control de esas enfermedades, teniendo como una de las estrategias públicas para la promoción de la alimentación sana. En este contexto, el objetivo de este trabajo fue evaluar la legislación brasileña vigente en cuanto al cuadro de profesional nutricionista para elaborar y acompañar el menú de la alimentación escolar, conforme prevé la Ley Federal n 8.234, de 17 de septiembre de 1991, y la Resolución del Consejo Federal de Nutricionistas - CFN n ${ }^{\circ}$ 380/2005. Se realizó el levantamiento de las Leyes que establecen un cuadro de profesionales nutricionistas en las escuelas públicas de las principales ciudades brasileñas en los órganos competentes. Los resultados muestran que pocos Estados y Municipios abordan ese tema, entre ellos el Estado de Río de Janeiro, el Estado de São Paulo, la ciudad de Ribeirão Preto, la ciudad de Florianópolis, la ciudad de Belém, el Estado de Santa Catarina, El Estado de Paraná, el Distrito Federal y el Estado de Ceará ya regularon la materia, así como la Ley n 13.666 / 2018 que modifica la Ley no 9.394 / 16. Los autores concluyen que además de conocer las pecurialidades de las manifestaciones orales de estos pacientes es imprescindible el conocimiento multidisciplinario del cirujano dentista, creando no sólo oportunidades de diagnóstico precoz, sino también la prevención y promoción de la salud oral.

PALABRAS CLAVE: salud pública, odontología, nutrición.

\section{INTRODUCCIÓN}

En Brasil, la prevalencia de enfermedades crónicas, que produce manifestaciones orales y que pueden interferir en la conducta del profesional de Odontología y en el establecimiento del plan de tratamiento, viene aumentando. Estos cambios en el perfil se caracterizan como un problema de salud pública de- mostrando que las principales causas de las enfermedades están asociadas a la alimentación inadecuada (de Moraes et al., 2014; Andrade et al., 2015; Didari et al., 2015; Distrutti et al., 2016; Enck et al., 2017; Revoredo et al., 2017; Mazzawi \& El-Salhy, 2017; Devanarayana \& Rajindrajith, 2018).

\footnotetext{
${ }^{1}$ Odontología pela UNINASSAU, Recife, Brasil.

2 Odontología pela ESAMAZ, Belem, Brasil.

${ }^{3}$ Odontología pela UFPA, Belem, Brasil.

${ }^{4}$ Medicina Tropical pela UFPA, Belem, Brasil.
} 
TEIXEIRA, J. R.; FARIAS, M. G.; CAMPOS, J. R.; SANTOS, R. K.; ALMEIDA, T.; REIS, A. C.; MARANHÃO, P. \& MARANHÃO, K. Estudio de la legalidad brasileña de las escuelas públicas municipales en cuanto a la promoción de la alimentación sana en el control y prevención a la salud oral en el contexto de las Enfermedades Gastrointestinales Inflamatorias. Int. J. Odontostomat., 13(3):363-366, 2019.

Las enfermedades gastrointestinales inflamatorias son una expresión generalmente utilizada para definir un grupo de enfermedades inflamatorias intestinales que se dividen en la enfermedad de Crohn, la colitis ulcerosa y la enfermedad celíaca. Los países económicamente desarrollados, así como la mayoría de los países en desarrollo, están actualmente definiendo estrategias para el control de esas enfermedades, teniendo como una de las estrategias la promoción de la alimentación sana, que es un componente importante en la prevención de estos problemas y en la educación alimentaria y nutricional, siendo por lo tanto una estrategia fundamental de intervención en el marco mencionado (Didari et al.; Revoredo et al.; Mazzawi \& El-Salhy; Devanarayana \& Rajindrajith; Protásio et al., 2018).

En este contexto, se hace evidente la necesidad de acciones para la socialización del conocimiento. De esta forma, el objetivo de este trabajo fue evaluar la legislación brasileña vigente en cuanto al cuadro de profesional nutricionista para elaborar, acompañar y evaluar el menú de la alimentación escolar, con base en el diagnóstico y en las referencias nutricionales, conforme prevé la Ley Federal $n^{\circ} 8.234$, de 17 de septiembre de 1991, y la Resolución del Consejo Federal de Nutricionistas - CFN n 380/2005.

\section{MATERIAL Y MÉTODO}

Se realizó el levantamiento de las Leyes que establecen un cuadro de profesionales nutricionistas en las escuelas públicas de las principales ciudades brasileñas en los órganos competentes. En seguida, estas fueron organizadas conforme a la jerarquía del sistema jurídico, que utiliza una pirámide en cuyo ápice está la Constitución (que es la ley máxima) y, debajo de ella, están las Leyes Complementarias, las Leyes Ordinarias y los Reglamentos. A partir de los resultados obtenidos, a través de la lectura fueron seleccionados solamente a aquellas que tratase de la Ley, la cual orienta que las redes públicas y privadas de enseñanza mantengan en cada unidad escolar un profesional del área de nutrición.

\section{RESULTADOS Y DISCUSIÓN}

Hay una relación fundamental entre el intestino y la salud, y dentro de la evaluación del proceso alimenticio, la eficaz absorción nutricional puede ser al- terada por un desequilibrio de la microbiota intestinal. Los factores que influyen en la formación de las enfermedades gastrointestinales inflamatorias, tales como las poblaciones bacterianas del intestino y la salud de la mucosa intestinal, están en íntegra relación con la nutrición del individuo. Así, la alimentación adecuada es un derecho fundamental del ser humano, reconocido internacionalmente por la declaración universal de los derechos humanos (Art. 25) y por el pacto internacional de derechos económicos, sociales y culturales - PIDESC (Art. 11), siendo inherente a la dignidad de la dignidad persona humana e indispensable para la realización de los derechos consagrados en la Constitución de la República. La Constitución Federal en su Art. 6 preconiza que son "derechos sociales la educación, la salud, la alimentación, el trabajo, la vivienda, el ocio, la seguridad, la seguridad social, la protección a la maternidad y la infancia, desamparados, en la forma de esta constitución (Revoredo et al.; Devanarayana \& Rajindrajith).

Los resultados muestran que pocos Estados y Municipios abordan ese tema en cuanto a la promoción de la alimentación sana, entre ellos se encuentran: el Estado y la ciudad de Río de Janeiro (Ley Estadual 7846/18; Decreto Estatal $n^{\circ}$ 4508/2005; Decreto Municipal $n^{\circ} 21217$ De acuerdo con lo establecido en la Ley Orgánica 15/1999 de la Comisión de las Comunidades Europeas. la ciudad de Florianópolis (Ley Municipal $n^{\circ} 5.853$ / 2001), la ciudad de Belém (DECRETO Nº 61.126 / 09, Ley municipal n8892 / 11), el Estado de Santa Catarina (Ley Estatal no 12.061 / 2001), el Estado de Paraná (En lo que se refiere a la Ley Orgánica del Poder Legislativo), el Estado de Ceará (Ley Estatal Ley $n$ ○ 16403/2017) ya reguló la materia, así como la Ley n 13.666 / 2018 que modifica la Ley nº 9.394 / 16.

Los pacientes con enfermedades inflamatorias intestinales pueden sufrir alteraciones de la mucosa oral. Entre las de mayor incidencia están ulceraciones aftosas, granulomatosas, reducción salivar, halitosis, mucogengivitis, queilitis angular, candidiasis y disfagia. La pioestomatitis vegetal se considera un marcador específico de la colitis ulcerativa por diversos autores. es una lesión idiopática constituida por múltiples pústulas con una base eritematosa y edemaciada. Las pústulas pueden romperse y unirse, formando úlceras lineales irregulares. Estas pústulas pueden alcanzar casi toda la cavidad oral, así como en el pavimento y las caras ventrales y laterales de la lengua (Cerchiari et al., 2006; Torres et al., 2011; de Mattos et al., 2014; Paixão \& Castro, 2016). 
TEIXEIRA, J. R.; FARIAS, M. G.; CAMPOS, J. R.; SANTOS, R. K.; ALMEIDA, T.; REIS, A. C.; MARANHÃO, P. \& MARANHÃO, K. Estudio de la legalidad brasileña de las escuelas públicas municipales en cuanto a la promoción de la alimentación sana en el control y prevención a la salud oral en el contexto de las Enfermedades Gastrointestinales Inflamatorias. Int. J. Odontostomat., 13(3):363-366, 2019.

En la Colitis ulcerosa, las manifestaciones orales se revelan menos comunes en comparación con lo que se observa en la enfermedad de Crohn (DC), patologías representativas de la enfermedad inflamatoria intestinal (DII). Algunos estudios reportan una mayor susceptibilidad a la enfermedad periodontal en pacientes con DII. En cuanto a la caries, parece consensual la existencia de un aumento de esta enfermedad en individuos con DII. Estas constataciones fueron justificadas de diversas formas, sin embargo, la dieta rica en azúcares corresponde a uno de los factores causales más señalados. Los pacientes celíacos presentaron defectos de desarrollo de esmalte, siendo éstos la opacidad demarcada y la opacidad difusa. Aunque la muestra es reducida, se puede inferir que esta prevalencia corrobora con los hallazgos de la literatura. Esta relación es fundamentada en la posible hipocalcemia durante la odontogénesis la cual es ocasionada por la mala absorción generada por la intolerancia al gluten que se encuentra presente en la mayoría de los los alimentos (Cerchiari et al.; Torres et al.; de Mattos et al.; Paixão \& Castro).

En la actualidad se ha discutido la creciente evidencia del papel del tracto gastrointestinal asociado con la alteración de la microbiota intestinal, en la aparición de los dolores crónicos musculoesqueléticos, entre ellos el Síndrome doloroso miofascial (SDM) y la fibromialgia (Brioschi et al., 2009). Las evidencias actuales afirman que las citocinas proinflamatorias contribuyen a este aumento de la nocicepción y que el estrés puede elevar la expresión de citocinas en el colon (IL-1b e IFNgama) (Bradesi et al., 2006). El consumo alimentario inadecuado puede exacerbar el proceso de transmisión de los impulsos dolorosos y potenciar la inflamación y, como consecuencia de ese consumo, el desequilibrio de la microbiota intestinal es un factor frecuente en los pacientes en tratamiento del dolor crónico miofascial, asociado al uso constante de medicamentos para control de la enfermedad (Brioschi et al.).

La identificación de estas lesiones requiere un examen sistemático de la cavidad oral, por lo que a menudo no se diagnostican. Incluso en gastroenterólogos familiarizados con estas manifestaciones, no siempre se efectúa un correcto diagnóstico. Así, una colaboración entre el médico Gastroenterólogo y el Médico Dentista permite un mejor diagnóstico de estas lesiones.

En la mayoría de los casos las manifestaciones orales aparecen meses o años después de la implicación intestinal, sin embargo, en ciertos casos pueden ser la primera manifestación, permitiendo un diagnóstico precoz. Las lesiones pueden ocurrir en cualquier momento durante el proceso de la enfermedad y pueden manifestarse de formas diferentes en el mismo paciente.

De esta forma, la alimentación puede ser considerada como uno de los factores comportamentales que más influye en la calidad de vida de las personas. La alimentación precede el proceso de la nutrición, que engloba las transformaciones que sufren los alimentos en el organismo vivo, comprendiendo así, digestión, absorción, utilización de energía y nutrientes (metabolismo) y reaprovechamiento o eliminación de subproductos de catabolismo. Sin embargo, la ingesta del alimento no garantiza que sus nutrientes serán biodisponibles para ser utilizados por las células. Para que esto realmente ocurra, es fundamental el equilibrio entre la cantidad y la calidad. Así, si una de esas etapas no funciona satisfactoriamente, el cuerpo presentará carencias nutricionales y funcionales. Es necesario nutrir el organismo adecuadamente, es decir, orientar la ingesta adecuada de alimentos, en cantidad y calidad, y aún, por medio de un hábito alimenticio correcto, garantizar que estos alimentos sean bien digeridos, absorbidos y utilizados. Por lo tanto, la prevención y el tratamiento de las DII pasa, principalmente, por una reeducación alimentaria. Así, la alimentación escolar sana actúa positivamente no sólo en el rendimiento escolar del alumno, como también, y principalmente en la promoción de la salud oral y prevención de enfermedades orales (Aragon et al., 2010; Rosa et al., 2014; Sinagra et al., 2017).

Se concluye que además de conocer las pecurialidades de las manifestaciones orales de estos pacientes es imprescindible el conocimiento multidisciplinario del cirujano dentista, creando no sólo oportunidades de diagnóstico precoz sino también la prevención y promoción a la salud oral.

TEIXEIRA, J. R.; FARIAS, M. G.; CAMPOS, J. R.; SANTOS, R. K.; ALMEIDA, T.; REIS, A. C.; MARANHÃO, P. \& MARANHÃO, K. Study of the Brazilian legality of the municipal public schools regarding the promotion of healthy eating for control and prevention of oral health in the context of inflammatory intestinal diseases. Int. J. Odontostomat., 13(3):363-366, 2019.

ABSTRACT: Inflammatory intestinal diseases are characterized by disharmony of the bacterial flora of the intestine. These changes are characterized as a public health problem demonstrating that the main causes of diseases are 
TEIXEIRA, J. R.; FARIAS, M. G.; CAMPOS, J. R.; SANTOS, R. K.; ALMEIDA, T.; REIS, A. C.; MARANHÃO, P. \& MARANHÃO, K. Estudio de la legalidad brasileña de las escuelas públicas municipales en cuanto a la promoción de la alimentación sana en el control y prevención a la salud oral en el contexto de las Enfermedades Gastrointestinales Inflamatorias. Int. J. Odontostomat., 13(3):363-366, 2019.

associated with inadequate nutrition and, as a consequence, changes in the oral mucosa. The developed countries are currently defining strategies for the control of these diseases, having as one of the public strategies to promote healthy eating. In this context, the objective of this study was to evaluate the Brazilian legislation in force regarding the professional dietitian to elaborate and follow the menu of school feeding, according to Federal Law No. 8,234, dated September 17, 1991, and the Resolution of the Federal Council of Nutritionists - CFN n ${ }^{\circ} 380 / 2005$. It was carried out the survey of the Laws that establish a cadre of nutrition professionals in the public schools of the main Brazilian cities in the competent bodies. The results show that few States and Municipalities address this theme, among them the State of Rio de Janeiro, the State of São Paulo, the city of Ribeirão Preto, the city of Florianópolis, the city of Belém, the State of Santa Catarina, State of Paraná, the Federal District and the State of Ceará have already regulated the matter, as well as Law 13,666 / 2018, which amends Law 9,394 / 16. The authors conclude that besides knowing the characteristics of the oral manifestations of these patients, a multidisciplinary knowledge of the dental surgeon is essential, creating opportunities for early diagnosis, as well as the prevention and promotion of oral health.

KEY WORDS: public health, dentistry, nutrition.

\section{REFERENCIAS BIBLIOGRÁFICAS}

Andrade, V. L. A.; Regazzoni, L. A. A.; Moura, M. T. R. S.; Anjos, E. M. S.; Oliveira, K. A.; Pereira, M.; Pereira, M. V. R.; Pereira, M. R. A.; Amorim, N. R. \& Iskandar, S. M. Obesidade e microbiota intestinal. Rev. Med. Minas Gerais, 25(4):583-9, 2015.

Aragon, G.; Graham, D. B.; Borum, M. \& Doman, D. B. Probiotic Therapy for Irritable Bowel Syndrome. Gastroenterol. Hepatol. (N. Y.), 6(1):39-44, 2010.

Bradesi, S.; Kokkotou, E.; Simeonidis, S.; Patierno, S.; Ennes, H. S.; Mittal, Y.; McRoberts, J. A.; Ohning, G.; McLean, P.; Marvizon, J. C.; et al. The role of neurokinin 1 receptors in the maintenance of visceral hyperalgesia induced by repeated stress in rats. Gastroenterology, 130(6):1729-42, 2006.

Brioschi, E. F. C.; Brioschi, M. L.; Yeng, L. T. \& Teixeira, M. J. Nutrição funcional no paciente com dor crônica. Rev. Dor, 10(3):276-85, 2009.

Cerchiari, D. P.; Moricz, R. D.; Sanjar, F. A.; Rapoport, P. B.; Moretti, G. \& Guerra, M. M. Síndrome da boca ardente: etiología. Rev. Bras. Otorrinolaringol., 72(3):419-24, 2006.

de Mattos, L. F. C.; dos Santos, C. M. M. L.; Roxo, M. A. P. \& Terezan, M. L. F. Possível associação entre doença periodontal e doenças intestinais inflamatórias crônicas. Braz. J. Periodontol., 24(3):5760, 2014.

de Moraes, A. C. F.; da Silva, I. T.; Almeida-Pititto, B. \& Ferreira, S. R. G. Microbiota intestinal e risco cardiometabólico: mecanismos e modulação dietética. Arq. Bras. Endocrinol. Metab., 58(4):317-27, 2014.

Devanarayana, N. M. \& Rajindrajith, S. Irritable bowel syndrome in children: Current knowledge, challenges and opportunities. World J. Gastroenterol., 24(21):2211-35, 2018.

Didari, T.; Mozaffari, S.; Nikfar, S. \& Abdollahi, M. Effectiveness of probiotics in irritable bowel syndrome: Updated systematic review with meta-analysis. World J. Gastroenterol., 21(10):3072-84, 2015.

Distrutti, E.; Monaldi, L.; Ricci, P. \& Fiorucci, S. Gut microbiota role in irritable bowel syndrome: New therapeutic strategies. World J. Gastroenterol., 22(7):2219-41, 2016.

Enck, P.; Aziz, Q.; Barbara, G.; Farmer, A. D.; Fukudo, S.; Mayer, E. A.; Niesler, B.; Quigley, E. M.; Rajilic-Stojanovic, M.; Schemann, M.; et al. Irritable bowel syndrome. Nat. Rev. Dis. Primers, 2:16014, 2016.

Mazzawi, T. \& El-Salhy, M. Effect of diet and individual dietary guidance on gastrointestinal endocrine cells in patients with irritable bowel syndrome (Review). Int. J. Mol. Med., 40(4):943-52, 2017.

Paixão, L. A. \& Castro, F. F. S. Colonização da microbiota intestinal e sua influência na saúde do hospedeiro. Universitas Ciênc. Saúde, 14(1):85-96, 2016

Protásio, B. K. P. F.; Barbosa, C. M. P. M. M.; Neufeld, C. B.; Buck, L. D.; Laund, L. S. L.; Toporovski, M. S. \& Visoni, T. C. Especificidades da apresentação da doença de Crohn na infância. Einstein (São Paulo), 16(1):eRC4070, 2018.

Revoredo, C. M. S.; Libânio, J.; Frazão, K. \& Cunha, M. Doença de Crohn e probióticos: uma revisão. RASBRAN Rev. Assoc. Bras. Nutr., 8(2):67-73, 2017.

Rosa, J. R.; Silva Júnior, J. F. \& Rosa, M. I. Perfil epidemiológico de portadores de doença inflamatória intestinal. A. C. M. Arq. Catarin. Med., 43(2):53-8, 2014.

Sinagra, E.; Morreale, G. C.; Mohammadian, G.; Fusco, G.; Guarnotta, V.; Tomasello, G.; Cappello, F.; Rossi, F.; Amvrosiadis, G. \& Raimondo, D. New therapeutic perspectives in irritable bowel syndrome: Targeting low-grade inflammation, immunoneuroendocrine axis, motility, secretion and beyond. World J. Gastroenterol., 23(36):6593-627, 2017.

Torres, J. A. P.; de Santana, R. M.; Torres, F. A. P.; Moura, A. R. \& Neto, J. R. T. Doenças inflamatórias intestinais no Hospital Universitário da Universidade Federal de Sergipe: manifestações extraintestinais. Rev. Bras. Colo-proctol., 31(2):115-9, 2011.

Dirección para correspondencia:

Ana Cássia Reis

Trav. São Francisco, 246 apto. 702.

PA CEP:66023-530

Bairro, Campina

Belém

BRASIL

Email: anacassiareis@gmail.com

Recibido : 16-12-2018

Aceptado: 12-03-2019 